\title{
Association of anti-apoptotic Mcl-1L isoform expression with radioresistance of oral squamous carcinoma cells
}

Vinayak C Palve and Tanuja R Teni

\begin{abstract}
Background: Oral cancer is a common cancer and a major health problem in the Indian subcontinent. At our laboratory Mcl-1, an anti-apoptotic member of the Bcl-2 family has been demonstrated to be overexpressed in oral cancers and to predict outcome in oral cancer patients treated with definitive radiotherapy. To study the role of Mcl-1 isoforms in radiation response of oral squamous carcinoma cells (OSCC), we investigated in the present study, the association of Mcl-1 isoform expression with radiosensitivity of OSCC, using siRNA strategy.

Methods: The time course expression of Mcl-1 splice variants (Mcl-1L, Mcl-1S \& Mcl-1ES) was studied by RT-PCR, western blotting \& immunofluorescence, post-irradiation in oral cell lines [immortalized FBM (radiosensitive) and tongue cancer AW8507 \& AW13516 (radioresistant)]of relatively differing radiosensitivities. The effect of Mcl-1L knockdown alone or in combination with ionizing radiation (IR) on cell proliferation, apoptosis \& clonogenic survival, was investigated in AW8507 \& AW13516 cells. Further the expression of Mcl-1L protein was assessed in radioresistant sublines generated by fractionated ionizing radiation (FIR).
\end{abstract}

Results: Three to six fold higher expression of anti-apoptotic Mcl-1L versus pro-apoptotic Mcl-1S was observed at mRNA \& protein levels in all cell lines, post-irradiation. Sustained high levels of $\mathrm{Mcl}-1 \mathrm{~L}$, downregulation of proapoptotic Bax \& Bak and a significant $(P<0.05)$ reduction in apoptosis was observed in the more radioresistant AW8507, AW13516 versus FBM cells, post-IR. The ratios of anti to pro-apoptotic proteins were high in AW8507 as compared to FBM. Treatment with Mcl-1L siRNA alone or in combination with IR significantly $(P<0.01)$ increased apoptosis viz. 17.3\% (IR), 25.3\% (siRNA) and 46.3\% (IR plus siRNA) and upregulated pro-apoptotic Bax levels in AW8507 cells. Combination of siRNA \& IR treatment significantly $(P<0.05)$ reduced cell proliferation and clonogenic survival of radioresistant AW8507 \& AW13516 cells, suggesting a synergistic effect of the Mcl-1L siRNA with IR on radiosensitivity. Interestingly, during the development of radioresistant sublines using FIR, high expression of Mcl-1L was observed.

Conclusion: Our studies suggest that $\mathrm{Mcl}-1 \mathrm{~L}$ isoform has an important role in the survival and radioresistance of OSCC and may be a promising therapeutic target in oral cancers.

\section{Introduction}

Squamous cell carcinoma of the oral cavity (OSCC) is the most prevalent cancer in males of the Indian subcontinent and is predominantly associated with the tobacco-chewing habit [1]. Radiotherapy is an important treatment modality in oral cancer aiding in tumor size reduction and preservation of oral function [2]. Despite advances in radiotherapy techniques, OSCC patients

\footnotetext{
* Correspondence: tteni@actrec.gov.in

Teni Lab, Advanced Centre for Treatment, Research and Education in Cancer (ACTREC), Tata Memorial Centre, Kharghar, Navi Mumbai 410210, India
}

frequently develop loco-regional recurrence resulting in 5 -year survival rates that have remained unchanged for past few decades [3]. Hence for successful radiotherapy, it is crucial to understand the mechanisms involved in the development of radiation resistance in tumor cells.

Anti-apoptotic members of the Bcl-2 family are the key regulators of cellular apoptosis and their over expression has been shown to be associated with radioresistance [4,5]. Mcl-1 (Myeloid cell leukemia-1), an anti-apoptotic member of the Bcl-2 gene family, is essential for development, differentiation, and proliferation

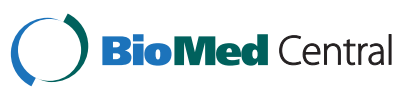


[6]. The overexpression of Mcl-1 has also been reported in a variety of hematopoietic, lymphoid and solid tumors [7-9]. Our earlier studies demonstrate the overexpression of anti-apoptotic Mcl-1 transcripts \& protein in oral tumors and cell lines [10]. Further, we have also demonstrated Mcl-1 to be a prognostic factor in oral cancer patients treated with definitive radiotherapy [11]. Earlier, Mcl-1 has been shown to contribute in resistance of cancer cells to chemotherapeutic agents, however reports on its role in radiation induced apoptosis and radioresistance are rare $[12,13]$. Further, the situation is complex due to the existence of distinct Mcl-1 isoforms having contrasting functions (anti-apoptotic Mcl-1L, pro-apoptotic Mcl-1S \& Mcl-1ES)[14]. Earlier studies from our lab have demonstrated a five to ten fold higher expression of anti-apoptotic Mcl-1L transcript, versus the pro-apoptotic Mcl-1S in oral tumors [10]. Therefore, in the present study we wanted to investigate the association of Mcl-1 isoforms with radioresistance of oral cancer cells using siRNA strategy. To the best of our knowledge, no reports are available on the role of Mcl-1 splice variants in radiation response of OSCC.

The present study was undertaken to compare the time course profile of Mcl-1 splice variants and other Bcl-2 family members, post-radiation (IR) treatment in oral cell lines of differing radiosensitivities. Further, the effect of Mcl-1L knockdown alone or in combination with IR on cell proliferation, apoptosis and radiosensitivity of oral cells was investigated.

\section{Materials and methods Cell culture}

Established AW8507 \& AW13516 [10,15] \& FBM (fetal buccal mucosa derived immortalized cell line) [16] were selected for the study due to their differing radiosensitivities. The cell lines were cultured in IMDM supplemented with 10\% FBS (Gibco, US), 100 units/ml penicillin, $100 \mu \mathrm{g} / \mathrm{ml}$ streptomycin, $2 \mathrm{mM}$ L-glutamine and keratinocyte growth supplements only for FBM (Sigma, USA), in $5 \% \mathrm{CO}_{2}$ at $37^{\circ} \mathrm{C}$.

\section{Clonogenic Assay}

Exponentially growing oral cells were harvested, counted and replated in duplicates. After $24 \mathrm{hrs}$, the cells were treated with different doses of IR $(1,2,4,6,8,10$ Gy) using ${ }^{60} \mathrm{Co}-\gamma$ radiator along with an untreated control. Cells were then incubated up to 14 days to form colonies which were fixed and stained with a mixture of glutaraldehyde $(6.0 \% \mathrm{v} / \mathrm{v})$ and crystal violet $(0.5 \% \mathrm{w} / \mathrm{v})$ and colonies ( $\geq 50$ cells) were counted using a microscope. The percent plating efficiency and fraction surviving a given radiation dose were calculated based on the survival of non-irradiated cells as described earlier [17].

\section{Radiation Treatment}

After $48 \mathrm{hrs}$ of plating exponentially growing cells $\left(6 \times 10^{3}\right.$ cells) were treated with IR $\left(D_{0}\right.$ dose $)$ using ${ }^{60} \mathrm{Co}-\gamma$ radiator as described earlier [13]. Cells were incubated upto different time points, harvested and stored in $-80^{\circ} \mathrm{C}$ until use.

\section{RNA isolation}

Cell pellets were placed in TRI reagent (Sigma, USA) and total cellular RNA was isolated according to the manufacturer's protocol. The RNA was dissolved in DEPC-treated water and contaminating DNA was removed by DNaseI treatment (Sigma, USA). RNA integrity was analyzed by electrophoresis and samples were preserved at $-80^{\circ} \mathrm{C}$ until analysis, as described earlier [10].

\section{Reverse transcriptase-polymerase chain reaction}

cDNA was synthesized with $2 \mu \mathrm{g}$ total RNA, using a First Strand cDNA synthesis kit (MBI Fermentas, Canada) according to the manufacturer's instructions. The efficiency of cDNA synthesis and equal loading were assessed by $ß$-actin PCR. Mcl-1 isoforms (Mcl-1L, Mcl-1S \& Mcl$1 \mathrm{ES}$ isoforms) were amplified by using primers (forward 5'ACGCGGTAATCGGACTCAACCT3' and reverse 5'GC AGCACATTCCTGATGCCACCT3'), as reported earlier [10]. A separate PCR was performed to determine expression of Mcl-1ES isoform using forward (5'ACGCGGTA ATCGGACTCAACCT3') and reverse (5'GCAGCACATT CCTGATGCCACCT3') primers as shown in Additional file 1 . PCR products were resolved on $2 \%$ agarose gel containing ethidium bromide and quantitated using Gel-doc system (UVP, UK).

\section{Western blotting}

Cell lysates were resolved on 12\% SDS-PAGE gels and transferred onto PVDF membranes (Millipore, USA). Membranes were blocked with 5\% skimmed milk in TBS for 2 hrs. Primary antibodies used were anti-Bax (1:150, Abcam, USA), anti-Mcl-1 (1:1000), anti-Bclxl (1:1000) and anti-ß-actin (1:2000) (Santa Cruz Biotechnology, USA). Secondary antibodies used were Horseradish peroxidase conjugated IgG (1:5000) (Santa Cruz Biotechnology, USA). Proteins were visualized with enhanced chemiluminescence kit (GE Healthcare, US). Densitometry analysis of developed X-ray film was performed using ImageJ software (NIH, Bethesda, MD). $\beta$-actin was used as loading control.

\section{Apoptosis detection by flow cytometry}

The Annexin V-FITC apoptosis detection kit (Santa Cruz Biotechnology, CA) was used for the detection of apoptotic cells in the three oral cell lines, as per the manufacturer's specifications. Briefly, cells were collected 
by trypsinization at different time points (Control, 1, 4, $24 \& 48 \mathrm{hrs}$ ) post-IR treatment. Cells were washed; $2 \mu \mathrm{g}$ Annexin-V FITC \& $10 \mu \mathrm{l}$ PI were added, incubated in the dark for $15 \mathrm{~min}$ and analyzed on a flow cytometer (FACS Caliber, BD, USA).

\section{Immunofluorescence staining}

Cells were grown on glass cover slips and Mcl-1 staining was performed at different time points in both FBM \& AW8507, post-IR using an Alexa fluor-488 labeled secondary antibody (1:1000, Molecular Probes, USA), as described earlier [10]. The AW8507 cells were treated with siRNA (100nM) and/or exposed to IR as described above. The nuclear condensation and apoptosis was analyzed by DAPI (Sigma-Aldrich, USA) staining, cell counting and imaging was done by confocal microscope with LSM Image Browser 4.2 software (Carl Zeiss).

\section{Knockdown of Mcl-1L isoform}

Knockdown was achieved using Mcl-1L specific siRNA (sc-43912) along with a control siRNA (sc-37007) from
Santa Cruz biotechnology, USA. The siRNA duplexes were transfected using Lipofectamine-2000 (Invitrogen) according to the manufacturer's instructions. The medium was changed after $16 \mathrm{hrs}$ of transfection and 24 hrs post-transfection the cells were assessed for knockdown by western blotting. The specific silencing of Mcl-1L was confirmed in three independent experiments.

\section{Trypan blue exclusion assay}

Cells were seeded into 24-well plates at a density of $5 \times 10^{4}$ per well and treated with Mcl-1L siRNA and/or IR as described above. Cells were trypsinized and trypan blue staining was performed after 48 hrs of treatment. The number of viable cells were counted and compared to untreated control using a hemocytometer.

\section{Acquired radioresistant sublines}

Radioresistant sublines were generated by irradiating AW8507 \& AW13516 cells with a fractionated Ionizing radiation (FIR) strategy as described earlier [18]. During development of radioresistant cell lines, cells were collected at different doses (Control, 8, 16, 24, 32, 40 Gy)

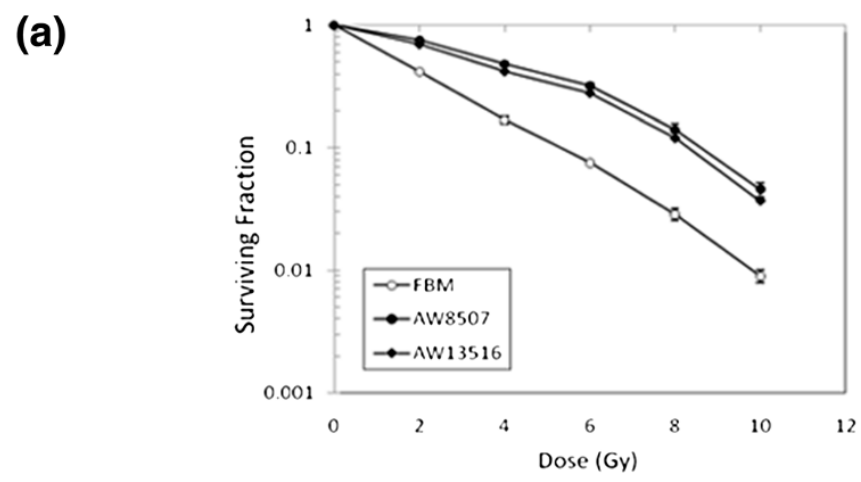

(b)
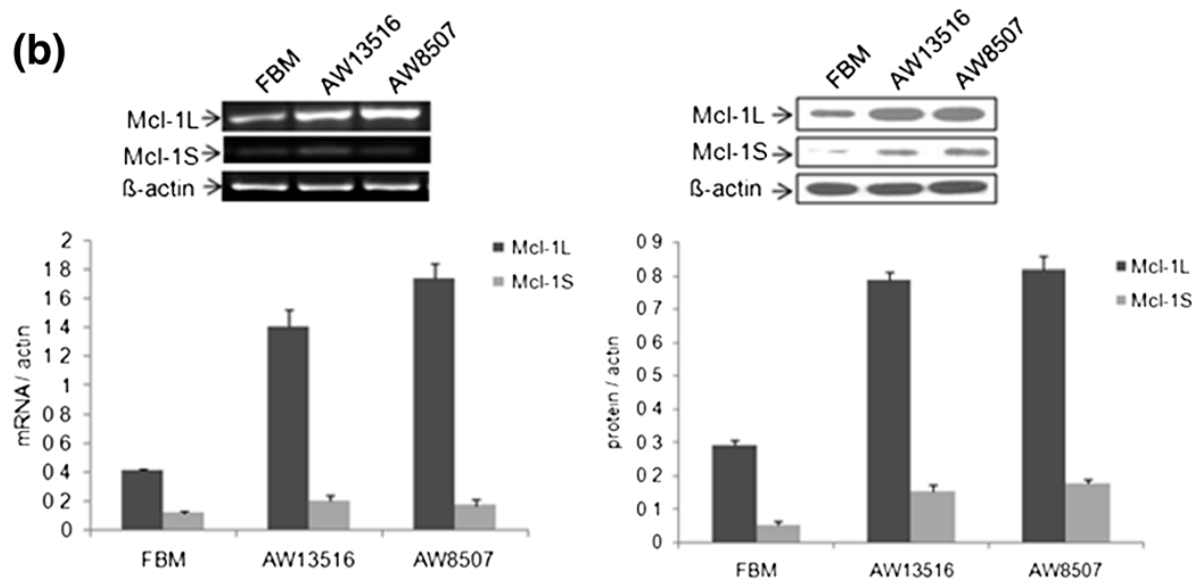

Figure 1 Radiosensitivity assessment and expression of Mcl-1 isoforms in oral cell lines. (a) Clonogenic cell survival assay of FBM, AW8507 \& AW13516 oral cell lines. Data given as percentage survival of untreated cell cultures and represent the means $( \pm$ SD) of three independent colony formation experiments. (b) Expression of Mcl-1 isoforms in oral cell lines. RT-PCR and western blot analysis of Mcl-1 isoforms in the three oral cell lines (FBM, AW13516 \& AW8507). A representative blot was shown for three independent experiments. Histogram indicates quantitative expression of $\mathrm{MCl}-1$ isoforms (MCl-1L \& MCl-1S) at both mRNA \& protein level in oral cell lines. 
and lysates from these sublines were loaded on SDS-PAGE to determine Mcl-1L expression by western blotting.

\section{Statistical analysis}

Statistical analysis was performed by using a Student's $t$-test analysis. The difference between means was considered statistically significant when $P<0.05$. The data is illustrated as mean \pm standard deviation of three independent experiments.

\section{Results}

Analysis of clonogenic survival of oral cell lines

Radiosensitivity assessment of the three oral cell lines (FBM, AW13516 \& AW8507) used in the study was determined by the clonogenic survival assay (Figure 1a). The $D_{0}$ values obtained from the surviving fractions of FBM, AW13516 \& AW8507 were 2.3, 5.1 \& 5.4 Gy respectively, indicating FBM to be most radiosensitive among all three oral cell lines.

\section{Expression of Mcl-1 splice variants in oral cell lines and effect of irradiation}

RT-PCR using a single primer which amplifies all three isoforms of Mcl-1 showed predominant expression of anti-apoptotic Mcl-1L and low levels of Mcl-1S but undetectable levels of Mcl-1ES in all three oral cell lines. Separate RT-PCR of poorly expressed Mcl-1ES isoform showed very low levels of Mcl-1ES as compared to Mcl-1L \& Mcl-1S in all the three oral cell lines (Additional file 1). The more radioresistant AW8507 \& AW13516 cells showed high expression of Mcl-1L at both mRNA \& protein levels as compared to immortalized FBM cells (Figure 1b). Post-IR, the time course expression profiles of Mcl-1 isoforms in the three oral cell lines revealed induction of Mcl-1L protein in all three cell lines. However, radiosensitive FBM exhibited a rapid and short induction profile with a peak at $1.5 \mathrm{hrs}$ which declined by $48 \mathrm{hrs}$. While the more radioresistant AW8507 exhibited sustained high levels of Mcl-1L up to $48 \mathrm{hrs}$ with a peak observed at $1.5 \mathrm{hrs}$ (Figure 2a). A similar pattern was observed in AW13516 (data not shown). In all cell lines, the expression of short Mcl-1S was elevated at initial time points which later decreased up to $48 \mathrm{hrs}$ while, the short pro-apoptotic Mcl-1ES isoform levels remained unaltered (See Additional file 2).

\section{Analysis of Bax, Bcl-xl \& Bcl-2 protein expression}

Interestingly, AW8507 cell line exhibited a rapid downregulation of pro-apoptotic Bax \& Bak proteins, $2 \mathrm{hrs}$ post-IR. In contrast the more radiosensitive FBM showed a consistent increase in Bax \& Bak levels, 2hrs onwards (Figure 2b). Higher expression of Bcl-2 \& Bcl-xl protein was observed in AW8507 as compared to FBM. The AW13516 cell line also showed similar results.

\section{Ratios of anti to pro-apoptotic members}

It is noteworthy that, the more radioresistant AW8507 cell line exhibited higher ratios of anti to pro-apoptotic proteins like Mcl-1L/Mcl-1S, Mcl-1L/Bax \& Bcl-xl/Bax as compared to that in FBM post-IR (Figure 3 a-f). A similar pattern was observed in AW13516 (data not shown).

\section{Effect of $\mathrm{Mcl}-1$ expression on apoptosis}

As compared to untreated control, post-IR up to $1 \mathrm{hr}$, a uniform time-dependent increase in apoptotic population was observed in all three oral cell lines. Notably, the number of apoptotic cells in AW13516 \& AW8507 significantly $(P<0.05$ at $24 \mathrm{hrs} \& P<0.01$ at $48 \mathrm{hrs})$ decreased thereafter as compared to that of FBM viz. from $17.5 \%$ to $9 \%$ at $24 \mathrm{hrs} \& 27 \%$ to $12 \%$ at $48 \mathrm{hrs}$ of post-IR (Figure 4a), coinciding with the high Mcl-1L/ Mcl-1S ratio (Figure 3b).

\section{Localization of Mcl-1 protein}

Immunofluorescence staining demonstrated that Mcl-1 protein was primarily localized in the cytosolic compartment of untreated AW8507 \& FBM cells. Interestingly post-IR a substantial increase in Mcl-1 protein expression with peri-nuclear and nuclear localization at $4 \mathrm{hrs}$ was observed in AW8507 cells, whereas no significant change in expression and localization was observed in FBM (Figure 4b).

\section{siRNA mediated downregulation of Mcl-1L}

AW8507 cells exhibited specific downregulation of Mcl1L levels after transfection with $100 \mathrm{nM}$ Mcl-1L siRNA without affecting the Mcl-1S levels (Figure 5a). The effect of Mcl-1L siRNA was maximal between 6 to $72 \mathrm{hrs}$ and the Mcl-1S levels were unaltered. Treatment with siRNA and IR alone or in combination significantly increased expression of pro-apoptotic Bax protein but did not change Bak \& Bcl-xl protein levels (Figure 5b).

\section{Effect Mcl-1L downregulation on cell proliferation and apoptosis}

Trypan blue dye exclusion assay in AW13516 \& AW8507 revealed a significant $(P<0.05)$ decrease in viability of cells treated with combination of siRNA plus IR as compared to individual treatments. After $72 \mathrm{hrs}$, cell viability was reduced to $67 \%$ (IR), $42 \%$ (siRNA) and $21 \%$ (IR plus siRNA) respectively (Figure 6a\&b). Thereby, suggesting a synergistic effect of the combined treatment on cell viability.

Immunofluroscence analysis of AW13516 \& AW8507 demonstrated an increased nuclear condensation in cells treated with combined Mcl-1L siRNA plus IR as compared to IR or siRNA alone (Figure 6c). The percentage of apoptotic cells in experimental control (EC), UC, IR, 


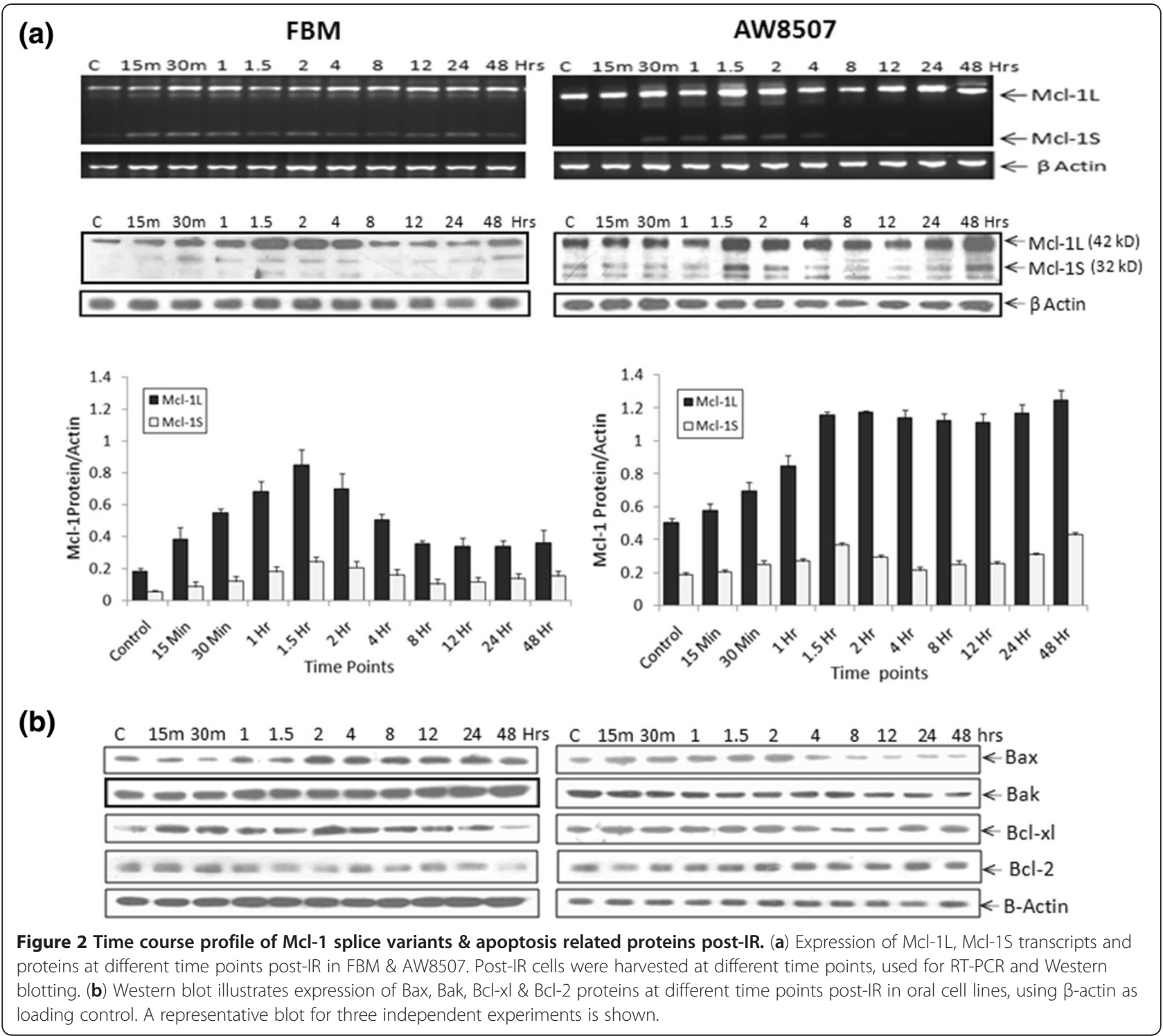

siRNA, siRNA plus IR treated AW8507 cells were 2.1\% (SD 0.5), 3.2\% (SD 0.6), 17.3\% (SD 0.5), 25.3\% (SD 1.1) and $46.3 \%$ (SD 0.6), respectively (Figure 6d). A similar pattern was observed in AW13516. The difference in percentage of apoptosis between IR alone and siRNA plus IR treated cells was highly significant $(P<0.01)$ in both the cell lines.

\section{Effect of Mcl1L knockdown on clonogenic survival}

The effect of Mcl-1L downregulation on long term cell survival was examined by clonogenic assay in AW8507 \& AW13516 cells. Interestingly, a reduction in clonogenic survival was observed after treatment of Mcl-1L siRNA (100nM) and increasing doses of IR as compared to the untreated control (Figure 7). The survival of AW8507 post-IR (0, 2, 4, 68 and 10Gy) was 78\% (SD 2.1), 46\%
(SD 1.5), 32\% (SD 2.3) and 14\% (SD 1.8) and 6\% (SD 1.9) respectively. However, in presence of $\mathrm{Mcl}-1$ siRNA the survival was further reduced to $42 \%$ (SD 2.6), 23\% (SD 1.7), $10 \%$ (SD 1.0), 4\% (SD 2.1) and 2\% (SD 1.7), respectively (Figure 7a). Similar reduction in clonogenic survival post Mcl-1L knockdown was observed in AW13516 cells (Figure 7b). These observations therefore suggest a synergistic effect of the Mcl-1L siRNA with IR on radiosensitivity.

\section{Expression of $\mathrm{Mcl}-1 \mathrm{~L}$ in radioresistant sublines}

To evaluate the association of $\mathrm{Mcl}-1 \mathrm{~L}$ with radioresistance, its expression was assessed by western blotting in acquired radioresistant sublines of AW8507 \& AW13516. Figure 8 demonstrates the high Mcl-1L expression in 


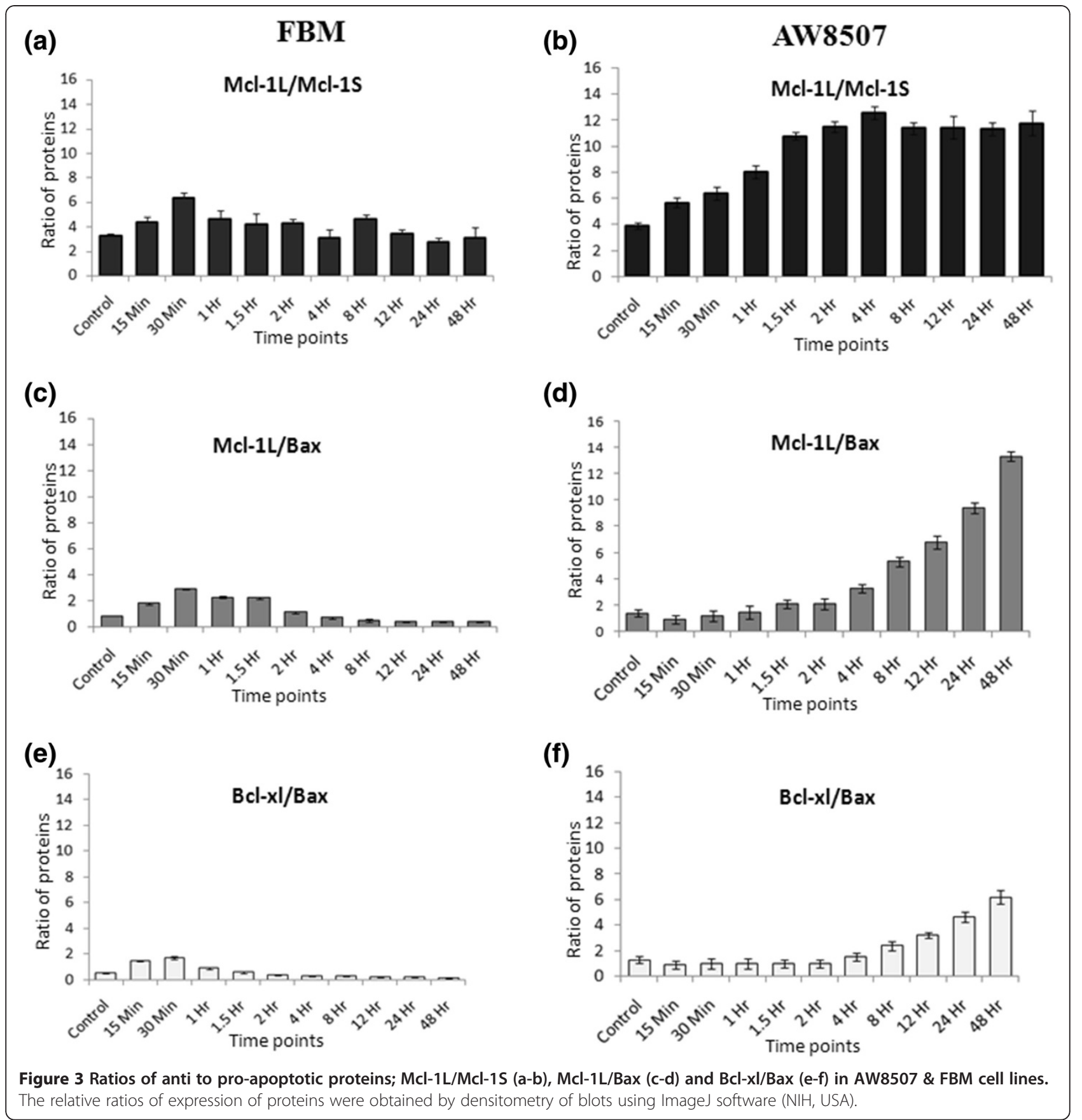

radio-resistant sublines generated by fractionated irradiation as compared to parental untreated cells.

\section{Discussion}

In the present study, we demonstrate the effect of antiapoptotic Mcl-1L expression on radiosensitivity of oral cancer cells. So far, limited information is available on the role of $\mathrm{Mcl}-1$ in radiation response of tumor cells. To our knowledge, this is the first study to report a time course expression of Mcl-1 isoforms post-IR and effect of Mcl-1L knockdown on radiosentitzation of oral cancer cell lines using siRNA strategy. Our studies demonstrated an inverse correlation of Mcl-1 expression with cellular apoptosis and a synergistic effect of Mcl-1L knockdown along with IR on cell viability and clonogenic survival thereby enhancing the radiosensitivity of OSCC cells.

Various growth factors and cellular stresses like radiation and cytotoxic agents are known to upregulate Mcl1 levels, thereby enhancing short term viability [19]. Our 
(a)

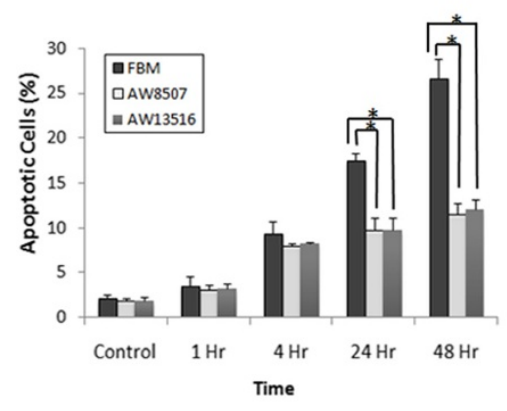

(b)

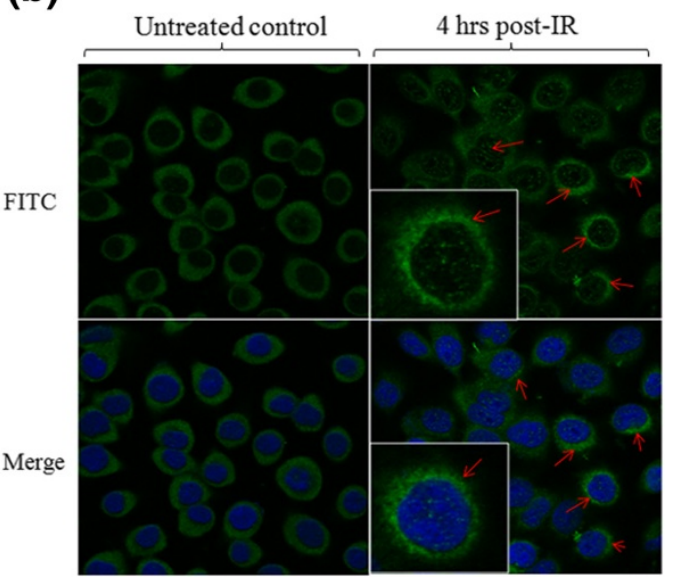

Figure 4 Apoptosis induction and localization of $\mathbf{M c l}-\mathbf{1}$ post-IR. (a) Percentage of apoptosis induction at different time points post-IR by Annexin- $V$ \& PI staining analyzed by FACS. The flow cytometry data shown is representative of three independent experiments ( ${ }^{*}<<0.05$ \& ${ }^{*} \mathrm{P}<0.01$ ) (b) Immunofluorescence staining of Mcl-1 protein counterstained with DAPI (blue) shows peri-nuclear accumulation (inset) and additional nuclear localization 4 hrs post-IR in AW8507 cells.

earlier studies had demonstrated higher expression of Mcl-1L transcript and its association with poor disease free survival in patients treated with definitive radiotherapy $[10,11]$. In the present study, two tongue cancer (AW8507 \& AW13516) and an immortalized oral (FBM) cell line were used due to their differing radiosensitivities and based on their $\mathrm{D}_{0}$ values, both AW8507 \& AW13516 were relatively more radioresistant than FBM. Therefore, to evaluate the association of Mcl-1L with radioresistance if any, we evaluated the expression of Mcl-1 isoforms in radioresistant AW8507 \& AW13516 as compared to radiosensitive FBM. Our studies revealed higher expression of Mcl-1L at both mRNA and protein level in relatively more radioresistant AW8507 \& AW13516 cell lines versus FBM, indicating a possible association of antiapoptotic Mcl-1L splice variant with radioresistance.

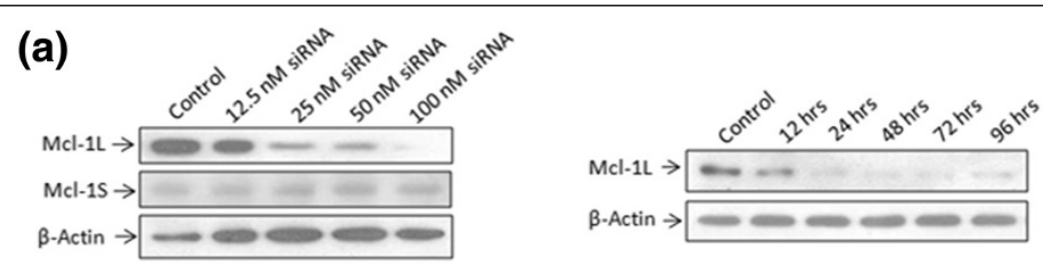

(b)
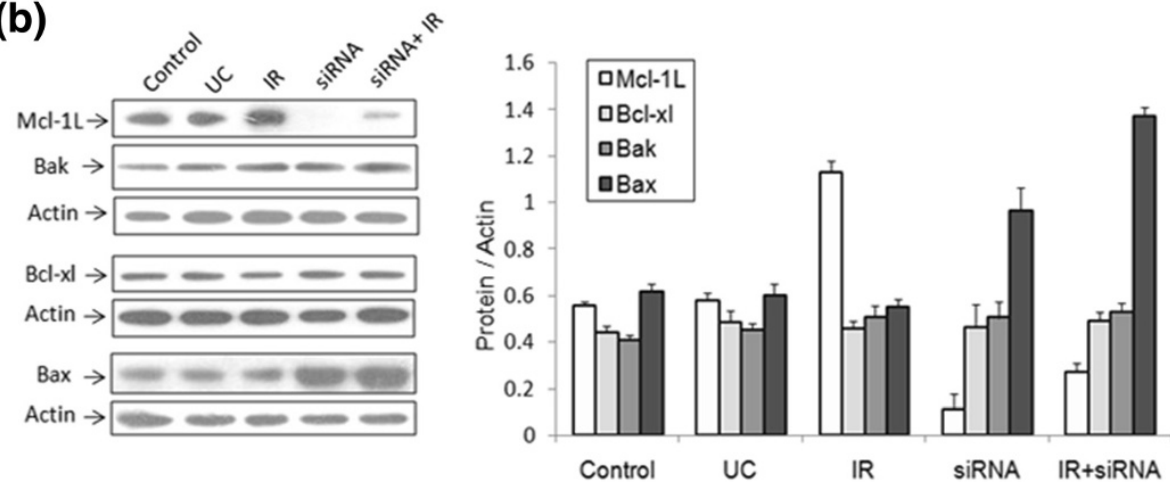

Figure 5 Western blot analysis of Mcl-1L knockdown. (a) MCl-1L downregulation using different concentrations of siRNA and unaltered expression of Mcl-1S in AW8507 cells. The effect of Mcl-1L siRNA was analyzed upto 96 hrs post transfection. (b) Expression of Mcl-1L, Bak, Bax \& BCl-xl proteins 24 hrs after transfection of AW8507 cells: [Experimental control without siRNA (EC); universal control siRNA (UC); MCl-1L siRNA (siRNA); irradiation (IR)]. A representative blot of three independently performed experiments is shown. 


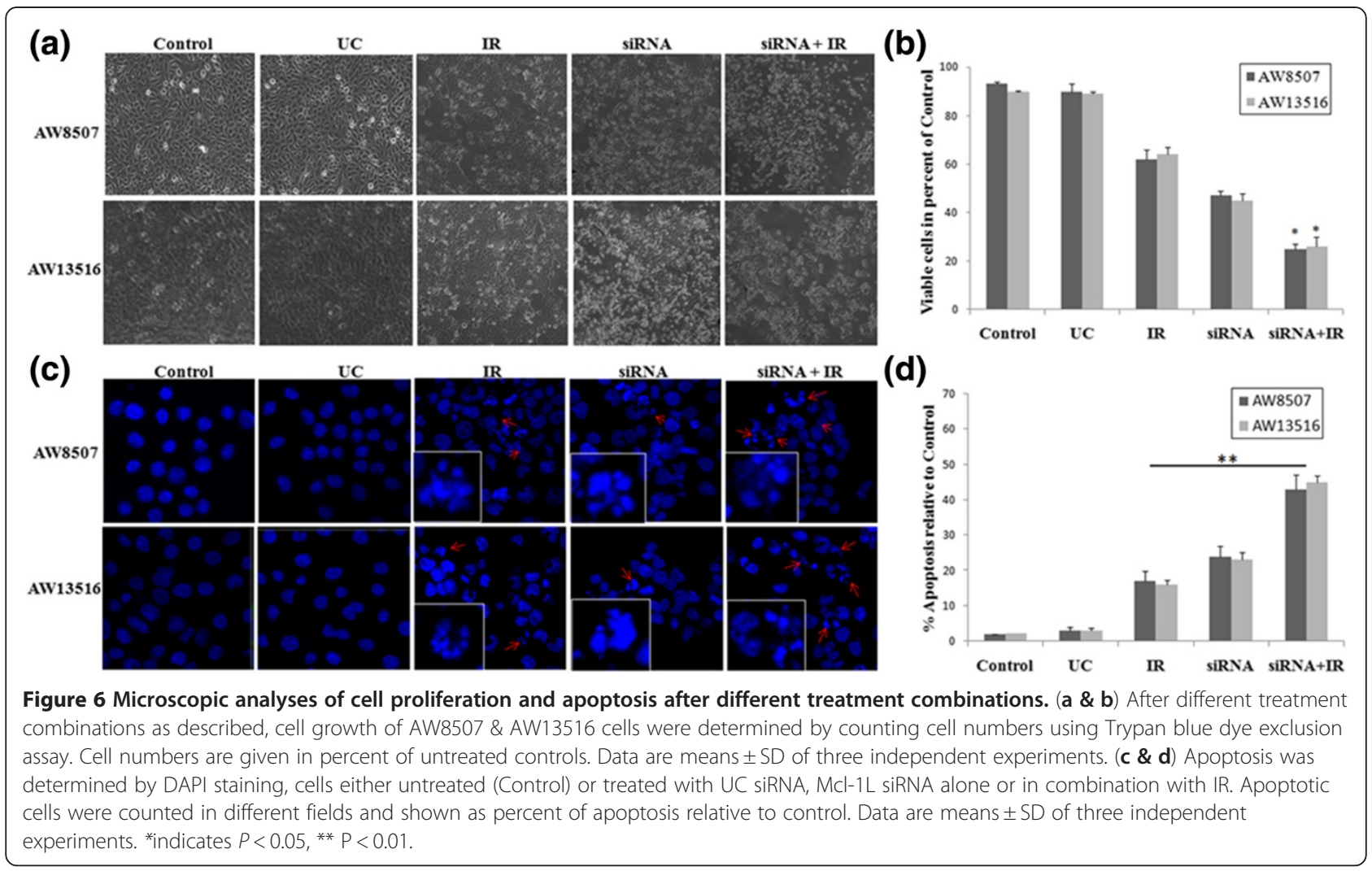

Several possible mechanisms can lead to the high Mcl-1 levels. Mcl-1 is known to be rapidly induced at the transcriptional level and its mRNA has a short half life [19]. Mcl-1 is also regulated at the post-transcriptional level by micro RNAs through a mir29 binding in the 3'UTR of Mcl-1 mRNA [20]. Interestingly, the expression of mir29 was also found to be decreased in malignant cholangiocytes, favoring the increased levels of Mcl-1, which indicates the possible reason for the observed high levels of Mcl-1 mRNA in our cancer cells lines and also responsible for its immediate degradation within few hours post IR.

Mcl-1, a PEST domain containing protein is also known to undergo ubiquitin dependent degradation by the $26 \mathrm{~S}$ proteosome and possesses a short half life of one to 3 hrs is rapidly downregulated during apoptosis (a)

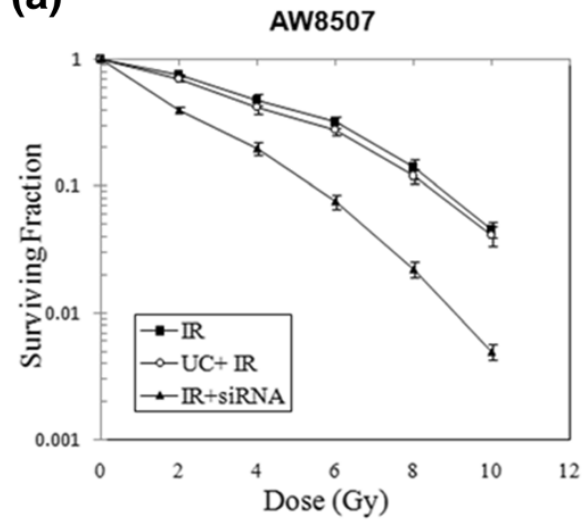

(b)

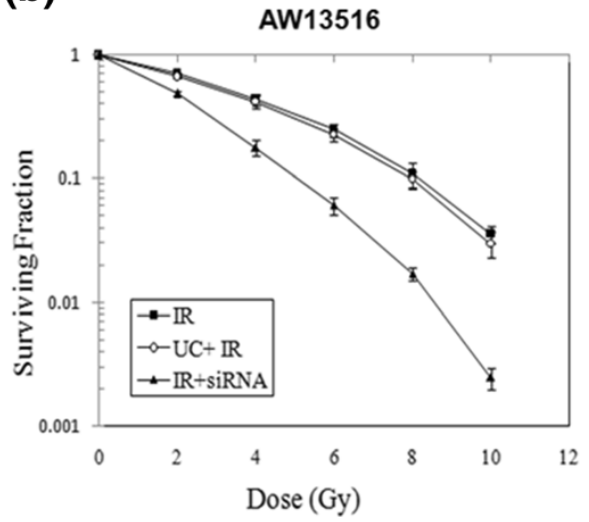

Figure 7 Clonogenic survival analysis after Mcl-1L knockdown and/or irradiation. (a \& b) AW8507 \& AW13516 cells were either untreated, or treated with siRNA. After $24 \mathrm{hrs}$, cell culture dishes were treated with increasing doses of ionizing irradiation (0, 2, 4, 68 and 10 Gy) as indicated. Survival was assessed by performing clonogenic assay as described in Methods. Data are given as percentage survival of untreated cells and represent the means $( \pm \mathrm{SD})$ of three independent colony formation experiments. 


\section{(a)

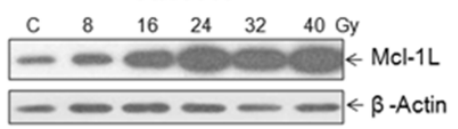

(b) AW13516

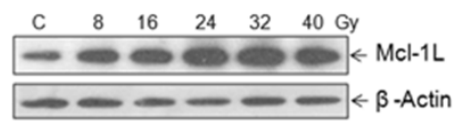

Figure 8 Expression of Mcl-1L in radio resistant sublines. (a \& b) AW8507 \& AW13516 cells were treated with fractionated irradiation and the radio resistant sublines were assayed for expression of Mcl-1L. $\beta$-actin blot from the same lysates serve as loading control.

[19]. Notably, the BH3 domains of MULE/LASU1 E3 ligase specifically interact with the hydrophobic BH3 binding pocket of $\mathrm{Mcl}-1$ and not with other antiapoptotic Bcl-2 family members [21] and are responsible for the constitutive turnover of Mcl-1. Such ubiquitin mediated degradation of Mcl-1 has been shown to be essential for the initiation of apoptosis, following UV damage [22]. Hence, it was interesting to study the time course expression profile of Mcl-1 isoforms and other bcl-2 family members in the above cell lines post-IR. Also, Mcl1 protein is known to be phosphorylated by GSK-3 $\beta$ at Ser159, located within the PEST domain, resulting in a significant decrease in the protein half-life and leading to initiation of apoptosis [23]. Such, alterations in the phosphorylation of Mcl-1 protein by GSK3 may also contribute to the elevation of Mcl-1 levels.

Moreover, it is known that the short isoform Mcl-1S only binds to $\mathrm{Mcl}-1 \mathrm{~L}$ possibly neutralizing its antiapoptotic function [24]. No significant alterations in levels of Mcl-1S \& Mcl-1ESwere observed post IR, indicating that the predominantly overexpressed $\mathrm{Mcl}-1 \mathrm{~L}$ isoform alone may contribute in generation of radioresistance. Mcl-1L not only binds to Mcl-1S, but is also known to heterodimerise with pro-apoptotic Bax \& Bak etc. preventing the release of cytochrome-c and subsequent apoptosis [25]. We observed a downregulation of pro-apoptotic Bax \& Bak proteins in AW8507 post-IR, coinciding with decreased apoptosis, while in contrast, FBM showed an increase in Bax \& Bak protein levels. We observed high expression of anti-apoptotic Bcl-xl which has already been shown to be associated with radiosensitization of colon cancer cells [26]. Thus, high expression of Bcl-xl \& Bcl-2, known radioresistant factors and $\mathrm{Mcl}-1 \mathrm{~L}$ in more radioresistant AW8507 \& AW13516 than FBM may indicate their possible role in radioresistance.

We assessed the ratios of Mcl-1L/Mcl-1S, Mcl-1L/Bax, Bcl-xl/Bax, wherein radioresistant AW8507\& AW13516 showed high ratios as compared to that in FBM indicating predominance of anti-apoptosis which may contribute to radioresistance. We are the first to elucidate the comparative levels of Mcl-1 isoforms and their association with radiation response in oral cell lines. The high and prolonged expression of Mcl-1L observed in AW8507 \& AW13516 could possibly be due to $\mathrm{Mcl}-1$ protein stabilization via binding with other proteins. Another reason could be its enhanced half life due to S159/T163 phosphorylation post-IR, reported to be crucial in nicotine mediated Mcl-1 activation and chemoresistance [27].

We observed a significant reduction in apoptosis postIR which coincided with the high levels of Mcl-1L, indicating a possible association of Mcl-1L expression with radiation response of AW8507 \& AW3516 cells. The immunofluorescence staining of $\mathrm{Mcl}-1$ indicated a perinuclear accumulation \& nuclear localization post IR in more radioresistant AW8507 cell line. Such, peri-nuclear accumulation of Mcl-1 has also been observed earlier in polymorphonuclear leukocytes post-etoposide treatment [28]. In AW8507, the observed nuclear and perinuclear accumulation of Mcl-1 may possibly help in cell survival to lower doses of DNA damaging agents. A similar regulatory role for $\mathrm{Mcl}-1$ (snMcl-1), perhaps acting as an adaptor protein in controlling the ATR-mediated regulation of DNA damage checkpoint kinase Chk1 phosphorylation and activation has been reported, placing Mcl-1 at the interface of apoptosis and cell cycle regulation [29]. Mcl-1 has been shown to regulate cell cycle by binding to proteins like CDK1 \& PCNA [30], possibly explaining the observed nuclear localization of Mcl-1. High expression of anti-apoptotic Mcl-1L and Bcl-xl proteins and reduced pro-apoptotic proteins like Bak \& Bax together may possibly contribute in lowering the sensitivity of AW8507 \& AW3516 cells to IR.

The downregulation of Mcl-1L alone was efficient in induction of apoptosis in both AW8507 \& AW13516 cells. Interestingly, the combination of Mcl-1L siRNA plus IR induced significantly higher apoptosis as compared to siRNA or IR-alone in both oral cell lines. Notably, the expression of closely related Bcl-xl, a known radioresistant factor was not altered. However, the expression of pro-apoptotic Bax protein correlated with the increased apoptosis on Mcl-1L knockdown. This overexpression of Bax, a downstream pro-apoptotic member, may execute the intrinsic apoptotic pathway resulting in increased cell death. To address the fact that the induction of apoptosis may not necessarily lead to long-term response to radiotherapy, we performed the clonogenic assay which demonstrated that combination of IR and Mcl-1L downregulation synergistically reduced 
clonogenic survival as compared to each treatment alone. Our studies demonstrate that Mcl-1L downregulation potentially enhanced radiosensitivity of AW8507 \& AW13516 cells in vitro. Complex interactions occur between $\mathrm{Bcl}-2$ family proteins especially, Bak \& Bax, where Mcl-1 plays a crucial role in engaging and maintaining pro-apoptotic Bak in an inactive state and accumulates $\mathrm{H} 2 \mathrm{AX}$ and ATM proteins to activate DNA repair pathways, suggesting that elimination of cellular Mcl-1 is crucial to initiate apoptotic pathway [31]. Overexpression and nuclear accumulation of Mcl-1 in AW8507 may occur due to a protein called IEX-1 which has been shown to interact specifically and timely with Mcl-1 controlling its accumulation and nuclear translocation in response to DNA damage and contribute in the activation of DNA repair pathway by Chk1 activation and G2 checkpoint arrest [32].

The high expression of Mcl-1L, in radioresistant sublines developed by fractionated ionizing radiation provides a direct evidence for the role of $\mathrm{Mcl}-1 \mathrm{~L}$ in radioresistance of OSCC cells. Therefore, the combination of radiotherapy and Mcl-1L downregulation has the potential to improve the response rate of treatmentresistant oral cancer cells. Selective inhibitors like Obatoclax, which specifically overcome Mcl-1 mediated resistance, is already in phase 2 clinical trials [33] and may have important therapeutic implications, when used in combination with radiotherapy in treatment of oral cancer patients.

\section{Conclusion}

Our studies indicate the association of Mcl-1L isoform expression with radioresistance by influencing apoptosis, proliferation and clonogenic survival of OSCC. Thus, Mcl-1L appears to be a promising molecular target for improving outcome of radiotherapy in oral cancer patients.

\section{Additional files}

Additional file 1: Figure S1. Indicating expression of Mcl-1ES isoform in three oral cell lines done by separate RTPCR.

Additional file 2: Figure S2. Time course profile of Mcl-1ES splice variant (done by separate PCR) along with another two (Mcl-1L \& Mcl-1S in single PCR) isoforms, at different time point's post-IR in AW8507 \& FBM cell lines.

\section{Abbreviations \\ Post-IR: Post Irradiation; FIR: Fractionated Ionizing Radiation; IR: Ionizing Radiation; IMDM: Iscove's Modified Dulbecco's Media; FBS: Fetal bovine serum; PBS: Phosphate-buffered saline; PVDF: Polyvinyldene fluoride; DAPI: 4'- 6-Diamidino-2-phenylindole; SD: Standard deviation; SDS-PAGE: Sodium dodecyle sulfate polyacrylamide gel electrophoresis.}

\section{Authors' contributions}

VP aided in study design, performed experimental procedures, analyzed the data and drafted manuscript. $\Pi$ designed the study and reviewed the manuscript. All authors read and approved the final manuscript.

Received: 10 May 2012 Accepted: 19 July 2012

Published: 8 August 2012

\section{References}

1. Tandle AT, Sanghvi V, Saranath D: Determination of p53 genotypes in oral cancer patients from India. Br J Cancer 2001, 84:739-742.

2. Burri RJ, Lee NY: Concurrent chemotherapy and radiotherapy for head and neck cancer. Expert Rev Anticancer Ther 2009, 9:293-302.

3. Mathew lype E, Pandey M, Mathew A, Thomas G, Krishnan Nair M: Squamous cell cancer of the buccal mucosa in young adults. $\mathrm{Br} J \mathrm{Oral}$ Maxillofac Surg 2004, 42:185-189.

4. Kyprianou N, King E, Bradbury D, Rhee J: bcl-2 over-expression delays radiation-induced apoptosis without affecting the clonogenic survival of human prostate cancer cells. Int J Cancer 1997, 70:341-348.

5. Wang ZB, Zhang Y, Liu YQ, Guo Y, Xu H, Dong B, Cui YF: Bcl-xL overexpression restricts gamma-radiation-induced apoptosis. Cell Biol Int 2006, 30:15-20.

6. Craig R: MCL1 provides a window on the role of the BCL2 family in cell proliferation, differentiation and tumorigenesis. Leukemia 2002, 16:444-454.

7. Sieghart W, Losert D, Strommer S, Cejka D, Schmid K, Rasoul-Rockenschaub S, Bodingbauer M, Crevenna R, Monia BP, Peck-Radosavljevic M, Wacheck V: $\mathrm{Mcl}-1$ overexpression in hepatocellular carcinoma: a potential target for antisense therapy. J Hepatol 2006, 44:151-157.

8. Derenne S, Monia B, Dean N, Taylor J, Rapp M, Harousseau J, Bataille R, Amiot M: Antisense strategy shows that $\mathrm{Mcl}-1$ rather than $\mathrm{Bcl}-2$ or $\mathrm{Bcl}-\mathrm{xL}$ is an essential survival protein of human myeloma cells. Blood 2002, 100:194

9. Cho-Vega $\mathrm{JH}$, Rassidakis GZ, Admirand $J \mathrm{H}$, Oyarzo M, Ramalingam $\mathrm{P}$, Paraguya A, McDonnell TJ, Amin HM, Medeiros $\sqcup$ : MCL-1 expression in B-cell non-Hodgkin's lymphomas. Hum Pathol 2004, 35:1095-1100.

10. Mallick S, Patil R, Gyanchandani R, Pawar S, Palve V, Kannan S, Pathak KA Choudhary M, Teni TR: Human oral cancers have altered expression of $\mathrm{Bcl}-2$ family members and increased expression of the anti-apoptotic splice variant of Mcl-1. J Pathol 2009, 217:398-407.

11. Mallick S, Agarwal J, Kannan S, Pawar S, Kane S, Teni T: PCNA and antiapoptotic $\mathrm{Mcl}-1$ proteins predict disease-free survival in oral cancer patients treated with definitive radiotherapy. Oral Oncol 2010, 46:688-693.

12. Guoan X, Hanning W, Kaiyun C, Hao L: Adenovirus-mediated siRNA targeting $\mathrm{Mcl}-1$ gene increases radiosensitivity of pancreatic carcinoma cells in vitro and in vivo. Surgery 2010, 147:553-561.

13. Skvara $H$, Thallinger $C$, Wacheck V, Monia BP, Pehamberger $H$, Jansen $B$, Selzer E: Mcl-1 blocks radiation-induced apoptosis and inhibits clonogenic cell death. Anticancer Res 2005, 25:2697-2703.

14. Kim JH, Sim SH, Ha HJ, Ko JJ, Lee K, Bae J: MCL-1ES, a novel variant of MCL-1, associates with MCL-1L and induces mitochondrial cell death. FEBS Lett 2009, 583:2758-2764.

15. Tatake RJ, Rajaram N, Damle RN, Balsara B, Bhisey AN, Gangal SG: Establishment and characterization of four new squamous cell carcinoma cell lines derived from oral tumors. J Cancer Res Clin Oncol 1990, 116:179-186.

16. Raul U, Sawant $S$, Dange $P$, Kalraiya $R$, Ingle A, Vaidya M: Implications of cytokeratin $8 / 18$ filament formation in stratified epithelial cells: induction of transformed phenotype. Int J Cancer 2004, 111:662-668.

17. Franken N, Rodermond H, Stap J, Haveman J, van Bree C: Clonogenic assay of cells in vitro. Nat Protoc 2006, 1:2315-2319.

18. Fukuda K, Sakakura C, Miyagawa K, Kuriu Y, Kin S, Nakase Y, Hagiwara A, Mitsufuji S, Okazaki Y, Hayashizaki Y, Yamagishi H: Differential gene expression profiles of radioresistant oesophageal cancer cell lines established by continuous fractionated irradiation. $\mathrm{Br} J$ Cancer 2004 91:1543-1550

19. Opferman JT: Unraveling MCL-1 degradation. Cell Death Differ 2006 , 13:1260-1262.

20. Mott JL, Kobayashi S, Bronk SF, Gores GJ: mir-29 regulates Mcl-1 protein expression and apoptosis. Oncogene 2007, 26:6133-6140. 
21. Warr MR, Acoca S, Liu Z, Germain M, Watson M, Blanchette M, Wing SS, Shore GC: BH3-ligand regulates access of MCL-1 to its E3 ligase. FEBS Lett 2005, 579:5603-5608.

22. Nijhawan D, Fang M, Traer E, Zhong Q, Gao W, Du F, Wang X: Elimination of $\mathrm{MCl}-1$ is required for the initiation of apoptosis following ultraviolet irradiation. Genes Dev 2003, 17:1475.

23. Maurer U, Charvet C, Wagman AS, Dejardin E, Green DR: Glycogen synthase kinase-3 regulates mitochondrial outer membrane permeabilization and apoptosis by destabilization of MCL-1. Mol Cell 2006, 21:749-760.

24. Bae J, Leo CP, Hsu SY, Hsueh AJ: MCL-1S, a splicing variant of the antiapoptotic BCL-2 family member MCL-1, encodes a proapoptotic protein possessing only the BH3 domain. J Biol Chem 2000, 275:25255-25261.

25. Thomas LW, Lam C, Edwards SW: Mcl-1; the molecular regulation of protein function. FEBS Lett 2010, 584:2981-2989.

26. Wacheck V, Selzer E, Gunsberg P, Lucas T, Meyer H, Thallinger C, Monia BP, Jansen $\mathrm{B}: \mathrm{BCl}-\mathrm{x}(\mathrm{L})$ antisense oligonucleotides radiosensitise colon cancer cells. Br J Cancer 2003, 89:1352-1357.

27. Zhao J, Xin M, Wang $T$, Zhang $Y$, Deng $X$ : Nicotine enhances the antiapoptotic function of Mcl-1 through phosphorylation. Mol Cancer Res 2009, 7:1954-1961.

28. Leuenroth SJ, Grutkoski PS, Ayala A, Simms HH: The loss of Mcl-1 expression in human polymorphonuclear leukocytes promotes apoptosis. J Leukoc Biol 2000, 68:158.

29. Jamil S, Sobouti R, Hojabrpour P, Raj M, Kast J, Duronio V: A proteolytic fragment of Mcl-1 exhibits nuclear localization and regulates cell growth by interaction with Cdk1. Biochem J 2005, 387:659.

30. Yang-Yen HF: Mcl-1: a highly regulated cell death and survival controller. J Biomed Sci 2006, 13:201-204.

31. Cuconati A, Mukherjee C, Perez D, White E: DNA damage response and MCL-1 destruction initiate apoptosis in adenovirus-infected cells. Genes Dev 2003, 17:2922-2932.

32. Pawlikowska P, Leray I, de Laval B, Guihard S, Kumar R, Rosselli F, Porteu F: ATM-dependent expression of IEX-1 controls nuclear accumulation of Mcl-1 and the DNA damage response. Cell Death Differ 2010, 17:1739-1750.

33. Paik PK, Rudin CM, Pietanza MC, Brown A, Rizvi NA, Takebe N, Travis W, James L, Ginsberg MS, Juergens R: A phase II study of obatoclax mesylate, $a$ $\mathrm{BCl}-2$ antagonist, plus topotecan in relapsed small cell lung cancer. Amsterdam, Netherlands: Lung cancer; 2011:74-481.

doi:10.1186/1748-717X-7-135

Cite this article as: Palve and Teni: Association of anti-apoptotic MCl-1L isoform expression with radioresistance of oral squamous carcinoma cells. Radiation Oncology 2012 7:135.

\section{Submit your next manuscript to BioMed Central and take full advantage of:}

- Convenient online submission

- Thorough peer review

- No space constraints or color figure charges

- Immediate publication on acceptance

- Inclusion in PubMed, CAS, Scopus and Google Scholar

- Research which is freely available for redistribution 